Wild, EJ; Tabrizi, SJ; (2007) The differential diagnosis of chorea. Practical Neurology , 7

(6) 360 - 373. 10.1136/pn.2007.134585.

\title{
ARTICLE
}

\section{The differential diagnosis of chorea}

Edward J Wild ${ }^{1}$ and Sarah J Tabrizi $i^{*}$

${ }^{1}$ Clinical Research Fellow and Honorary Clinical Assistant

${ }^{2}$ Department of Health Clinician Scientist and Honorary Consultant Neurologist

Department of Neurodegenerative Disease, UCL Institute of Neurology, London / National Hospital for Neurology and Neurosurgery, Queen Square, London

Word count: 3999

References: 40

${ }^{*}$ Corresponding author.

Disclosure: The authors report no conflicts of interest. 


\section{Introduction}

Chorea is a hyperkinetic movement disorder characterised by excessive spontaneous movements that are irregularly timed, randomly distributed and abrupt. It ranges in severity from restlessness with mild, intermittent exaggeration of gesture and expression, fidgety movements of the hands (seen online video) and unstable dance-like gait to a continuous flowing of disabling and violent movements. In this article we discuss the causes of chorea and an approach to its assessment.

\section{A brief history of chorea}

The term chorea is from the Greek koreia $(\chi 0 \rho \varepsilon 1 \alpha)$ meaning a dance. The word was first used medically by the alchemist Paracelsus (1493-1541) to describe Chorea Sancti Viti (St Vitus' dance), which was probably an epidemic form of hysterical chorea occurring in the context of religious or superstitious fervour. ${ }^{1}$ Thomas Sydenham (1624-1689), studying St Vitus' dance, identified a form of childhood chorea that now bears his name.

One year after obtaining his medical qualification, George Huntington (1850-1916) in 1872 described a hereditary form of chorea "spoken of by those in whose veins the seeds of the disease are known to exist, with a kind of horror", with onset in adult life and associated with cognitive and psychiatric manifestations. Huntington's disease (HD) is the commonest hereditary cause of chorea, and the mutation causing it was identified in 1993 as a result of an international collaborative initiative. ${ }^{2}$

\section{Differential diagnosis}

The major causes of chorea can be simply divided into inherited and acquired (Table 1).

\section{Table 1 Major inherited and acquired causes of chorea}

\section{Inherited causes}

- Huntington's disease* 
- Neuroacanthocytosis

- Macleod syndrome

- Dentatorubro-pallidoluysian atrophy (DRPLA)

- Benign hereditary chorea

- Spinocerebellar ataxia (SCA) types 1, 2, 3 and 17

- Mitochondrial disorders

- Inherited prion disease

- Huntington's disease-like 2 (HDL2)

- Wilson's disease

- Friedreich's ataxia

- Neurodegeneration with brain iron accumulation (NBIA)

- Ataxia telangiectasia

- Neuroferritinopathy

- Lysosomal storage disorders

- Amino acid disorders

- Tuberose sclerosis

\section{Acquired causes}

- Focal striatal pathology
- Stroke
- Space-occupying lesions

- Drug-induced (see Table 3)

- Chorea gravidarum

- Thyrotoxicosis

- Systemic lupus erythematosus (SLE) / Antiphospholipid syndrome (APLS)

- Post-infective

- Sydenham's chorea (group A streptococcal infection)

- Paediatric autoimmune disorders associated with streptococcal infections (PANDAS)

- Herpes simplex encephalitis

- Polycythaemia rubra vera

- Infective

- AIDS

- Variant Creutzfeld-Jakob disease (vCJD)

${ }^{*}$ the commonest cause of inherited chorea-the remainder of these disorders are rare.

\section{Inherited causes}

\section{Huntington's disease}

Huntington's disease (HD) is a progressive autosomal dominant neurodegenerative disorder and the commonest inherited cause of chorea. Onset is usually in adult life with a mean age of about 40 years, although juvenile and elderly onset are well described. It progresses inexorably, with death occurring 15-20 years from the time of onset. Prevalence is 4-10 per 100,000 in Western European populations, but HD has been described throughout the world. 


\section{Genetics and pathology}

The gene defect associated with HD is a CAG repeat expansion, encoding polyglutamine repeats within the huntingtin protein. ${ }^{2}$ The CAG repeat ranges between 10 and 29 copies on normal chromosomes, and is expanded to a range of $36-121$ on HD chromosomes. CAG repeats above 40 are fully penetrant, with a borderline range between 36 and 39 repeats associated with reduced penetrance. ${ }^{3}$

CAG repeat lengths tend to increase down generations, particularly when transmitted through the paternal lineage. ${ }^{4}$ This underlies the phenomenon of anticipation-the increasing severity of HD during intergenerational transmission. There is a correlation between CAG repeat size and age of onset-the larger the repeat, the earlier the disease onset. ${ }^{5}$ Most individuals with greater than 50 repeats develop the disease before the age of $30 .{ }^{6}$ However, CAG repeat length accounts for only $50-70 \%$ of variation of age of onset, and does not predict the clinical phenotype nor the severity of disease progression. ${ }^{7}$

\section{Motor features}

HD can produce a wide range of phenotypic presentations and the signs and symptoms change as it progresses. ${ }^{8}$

The onset of HD is often difficult to discern clearly and many patients have psychiatric problems or cognitive symptoms prior to the appearance of any motor problems. The definitive diagnosis of $\mathrm{HD}$ is usually made when motor abnormalities are noted on examination. Minor motor abnormalities seen early in the disease are general restlessness, abnormal eye movements, hyperreflexia and fidgety movements of fingers, hands and toes during stress or when walking. Oculomotor abnormalities are a cardinal feature of the disease and often the earliest motor sign. Saccadic abnormalities are characteristic, including delayed initiation and slowing and inability to suppress glances at novel stimuli. As the disease progresses, blinking and head thrusting are seen during saccade initiation. Later, impaired pursuit and gaze impersistence are seen. ${ }^{9}$ Patients develop more obvious 
extrapyramidal signs; chorea is seen in $90 \%$ of adult-onset patients with varying degrees of dystonia, parkinsonism and bradykinesia. A key motor abnormality in HD is impairment of voluntary motor function with clumsiness, motor impersistence, disturbances in fine motor control and motor speed. Gait disturbance is common with impairment of postural reflexes making patients more prone to falling. Dysarthria and dysphagia are common.

\section{Cognitive features}

Cognitive abnormalities are a near-universal feature and present early in the course of HD but vary greatly in severity. Executive function is most impaired with poor planning and judgement, impulsive behaviour, disorganisation and difficulty coping with multiple tasks. Many patients exhibit psychomotor slowing with apathy, lack of self-care and loss of initiative. $^{10}$

\section{Psychiatric features}

Psychiatric symptoms are common, particularly depression and anxiety. ${ }^{10}$ Irritability is also common and some patients become aggressive. Obsessions and compulsions can make life difficult for carers. Psychosis is well-recognised but rare. Suicide is much more common in HD in the general population. ${ }^{11}$ Psychiatric symptoms should be actively managed with the same drugs used in standard psychiatric practice. ${ }^{12}$

\section{Juvenile $H D$}

Juvenile HD cases have more severe disease with a shorter life expectancy. The akineticrigid form of the disease (Westphal variant) is more common in juvenile HD cases: patients have little chorea and are rigid, Parkinsonian and dystonic. Akinetic-rigid forms of the disease are rare in older age groups. Juvenile HD patients have a higher incidence of seizures than adult-onset patients. ${ }^{8}$ 


\section{Huntington's disease phenocopies}

The combination of chorea, cognitive impairment, psychiatric abnormalities and an autosomal dominant family history was diagnostic of HD until the advent of diagnostic genetic testing. $1 \%$ of suspected HD cases test negative for the CAG expansion and are known as HD phenocopies. The major genetic HD phenocopy syndromes are listed in Table 2. A diagnosis is made in only $2 \%$ of HD phenocopy patients. ${ }^{13}$

Table 2 Genetic causes of HD phenocopy syndromes

\begin{tabular}{ll}
\hline Condition & Cause \\
\hline HDL1 & Octapeptide insertion in gene encoding prion protein \\
HDL2 & Triplet repeat expansion in gene encoding junctophilin-3 \\
HDL3 & Causative mutation unidentified \\
SCA17 & Triplet repeat expansion in gene encoding TATA-box binding \\
& protein \\
Inherited prion disease & Mutations in gene encoding prion protein \\
SCA1 & Triplet repeat expansion in gene encoding ataxin-1 \\
SCA3 & Triplet repeat expansion in gene encoding ataxin-3 \\
DRPLA & Triplet repeat expansion in gene encoding atrophin-1 \\
Chorea-acanthocytosis & Mutation in gene encoding chorein \\
Neuroferritinopathy & Mutations in gene encoding ferritin light-chain \\
\hline
\end{tabular}

HDL1 is a rare inherited prion disorder. HDL2 is caused by a CAG/CTG expansion in the gene encoding junctophilin-3 and is generally rare, except in individuals of African ancestry where it is almost as common as HD. ${ }^{14}$

\section{Spinocerebellar ataxias}

The spinocerebellar ataxias (SCAs) are a group of autosomal dominant neurodegenerative conditions, causing cerebellar ataxia and a spectrum of associated features. SCAs may cause hyperkinetic movement disorders including chorea. SCA17, causes cognitive decline and neuropsychiatric features ${ }^{15}$ and is the commonest cause of HD phenocopy syndromes in European populations. ${ }^{13}$ Dentatorubral-pallidoluysian atrophy (DRPLA) causes ataxia, chorea and cognitive impairment but is usually distinguished from HD by the presence of myoclonic epilepsy. ${ }^{15}$ 


\section{Wilson's disease}

Wilson's disease is an autosomal recessive disorder of copper metabolism caused by mutations in ATP7B. Its neurological manifestations most commonly comprise an akineticrigid syndrome with dysarthria, dystonia, tremor and ataxia. $16 \%$ of patients experience chorea. Kayser-Fleischer rings in the cornea and MRI abnormalities are seen in almost all patients with neurological signs and the diagnosis is confirmed by low serum caeruloplasmin, high urinary copper levels and high copper concentration on liver biopsy. ${ }^{16}$

\section{Mitochondrial disease}

Mutations in DNA encoding mitochondrial proteins are increasingly recognised as causes of neurological disease. Myoclonus and dystonia are the most common movement disorder seen in mitochondrial disease but chorea can occur. ${ }^{17}$

\section{Iron accumulation disorders}

Neuroferritinopathy is a rare autosomal dominant disorder caused by mutations in FTL. It results in abnormal iron accumulation in the basal ganglia causing chorea and other movement disorders as well as cognitive impairment. ${ }^{18}$ Neurodegeneration with brain iron accumulation (NBIA), caused by mutations in PANK2, is an autosomal recessive cause of chorea. ${ }^{19} \mathrm{MRI}$ imaging is invariably abnormal in these disorders. ${ }^{18,19}$

\section{Neuroacanthocytosis}

Neuroacanthocytosis refers to a group of neurological disorders in which acanthocytes are seen on peripheral blood films. Chorea-acanthocytosis is a rare autosomal recessive disorder associated with mutations in $C H A C .^{20}$ It causes a progressive, adult-onset syndrome encompassing movement disorder, psychiatric and cognitive changes that may mimic HD (see Table 2). The movement disorder includes chorea, dystonia and tics. Orofacial dystonia is characteristic, with tongue protrusion and biting of the tongue and lips. Psychiatric and cognitive features are similar to HD. Unlike HD, seizures are seen in $50 \%$ of patients, and there is commonly distal amyotrophy or neuropathy with elevated serum 
creatine kinase.$^{21}$ Macleod syndrome is an X-linked recessive disorder linked to mutations in $X K$. Limb chorea is and facial tics are common but dystonia is less prominent than in chorea-acanthocytosis. Axonal neuropathy, subcortical dementia and psychiatric features are features. Cardiomyopathy and haemolytic anaemia are common. ${ }^{21}$

\section{Benign hereditary chorea}

Benign hereditary chorea $(\mathrm{BHC})$ is a dominantly inherited disorder caused by mutations in TITF1. It is usually young-onset with very slowly progressive chorea without cognitive decline or other neurological features. ${ }^{22}$ However, BHC may include atypical clinical features such as dystonia, myoclonus, dysarthria, gait disturbances and intellectual impairment. ${ }^{23}$

\section{Friedreich's ataxia}

Friedreich's ataxia, which is autosomal recessive, is usually characterised by progressive cerebellar ataxia, peripheral neuropathy, cardiac abnormalities and diabetes mellitus. Chorea is a rare manifestation. ${ }^{24}$

\section{Acquired causes of chorea}

A cause is identified in most cases of acquired chorea. In a recent Italian case series, the commonest causes were stroke, drug-induced, AIDS-related and metabolic (hyperglycaemia, hyponatraemia and hypoxia), ${ }^{25}$ though there is regional variation: in Brazil, for example, Sydenham's chorea is responsible for the majority of cases of acquired chorea. $^{26}$

\section{Vascular chorea}

Vascular lesions of the basal ganglia typically cause rapid-onset chorea affecting the contralateral limbs. Stroke-induced chorea often remits spontaneously. ${ }^{27}$

\section{Post-infective autoimmune CNS disorders}

Sydenham's chorea and PANDAS (paediatric autoimmune neuropsychiatric disorders associated with streptococcal infection) present with chorea and neuropsychiatric 
manifestations. Sydenham's chorea is one of the major manifestations of rheumatic fever. It occurs in children, mainly girls, and is now rare in the developed world. Chorea, tics, behavioural disturbance and obsessive-compulsive symptoms are common. It is self-limiting and usually resolves within 6 months, though about $20 \%$ of cases are recurrent. Anti-basal ganglia antibodies can be detected. ${ }^{28}$ The disease mechanism is thought to be crossreaction between anti-streptococcal antibodies and basal ganglia neurons. Treatment with steroids, intravenous immunoglobulin and plasmapheresis may be effective in refractory cases. $^{29,30}$ Chorea with anti-basal ganglia antibodies has been reported following herpes simplex encephalitis. ${ }^{31}$

\section{Drug-induced chorea}

Neuroleptic-induced tardive dyskinesia and levodopa-induced hyperkinesia are the commonest medication causes of chorea in neurological practice. Many drugs are known to cause chorea; the commonest causes are shown in Table 3. Most such reactions remit on ceasing the offending medication but some (notably tardive dyskinesia) may persist for months or years. ${ }^{32}$ Chorea induced by the oral contraceptive pill (OCP) is linked to childhood Sydenham's chorea and may be mediated by anti-basal ganglia antibodies. ${ }^{33}$

\section{Table 3. Medications most commonly causing chorea}

- Neuroleptics

- Levodopa

- Anticonvulsants

- Phenytoin

- Carbamazepine

- Valproate

- Gabapentin

- CNS stimulants

- Amphetamines

- Cocaine

- Methylphenidate

- Benzodiazepines

- Oestrogen-containing OCP

- Lithium

- Dopamine agonists 


\section{Other acquired causes}

$4 \%$ of patients with systemic lupus erythematosus (SLE) develop chorea. It usually occurs early in the disease and may precede other findings by several years. Patients with SLE who produce antiphospholipid antibodies are more likely to develop chorea. The antiphospholipid syndrome-the association of such antibodies and thrombosis, miscarriage and thrombocytopenia-may occur independently of SLE and is a cause of chorea in its own right. ${ }^{34}$ Chorea is a rare feature of thyrotoxicosis. It relates directly to elevated thyroid hormone levels and usually resolves once the euthyroid state is restored. ${ }^{35}$ Hemichorea is the movement disorder most commonly associated with AIDS, and can be the presenting feature. In one case series, $5 / 51$ cases of acquired chorea were AIDS-associated.$^{25}$ Chorea in AIDS is usually due to a striatal toxoplasmic abscess. Rarer causes of chorea in AIDS include cryptococcus, progressive multifocal leukoencephalopathy, HIV encephalitis and drug treatment. ${ }^{36}$ Chorea gravidarum usually presents in the first trimester of pregnancy and is self-limiting. Previous rheumatic fever, anti-basal ganglia antibodies and antiphospholipid syndrome are risk factors. ${ }^{37}$ Chorea is a rare feature of polycythaemia rubra vera, a myeloproliferativea disorder causing increased red cell mass that commonly produces neurological manifestations. ${ }^{38}$ Chorea is not seen in secondary polycythaemia, suggesting that it relates to an underlying molecular abnormality. ${ }^{39}$

\section{Assessment}

Assessment of the choreic patient depends on directed history, examination and investigations (figure 1). 


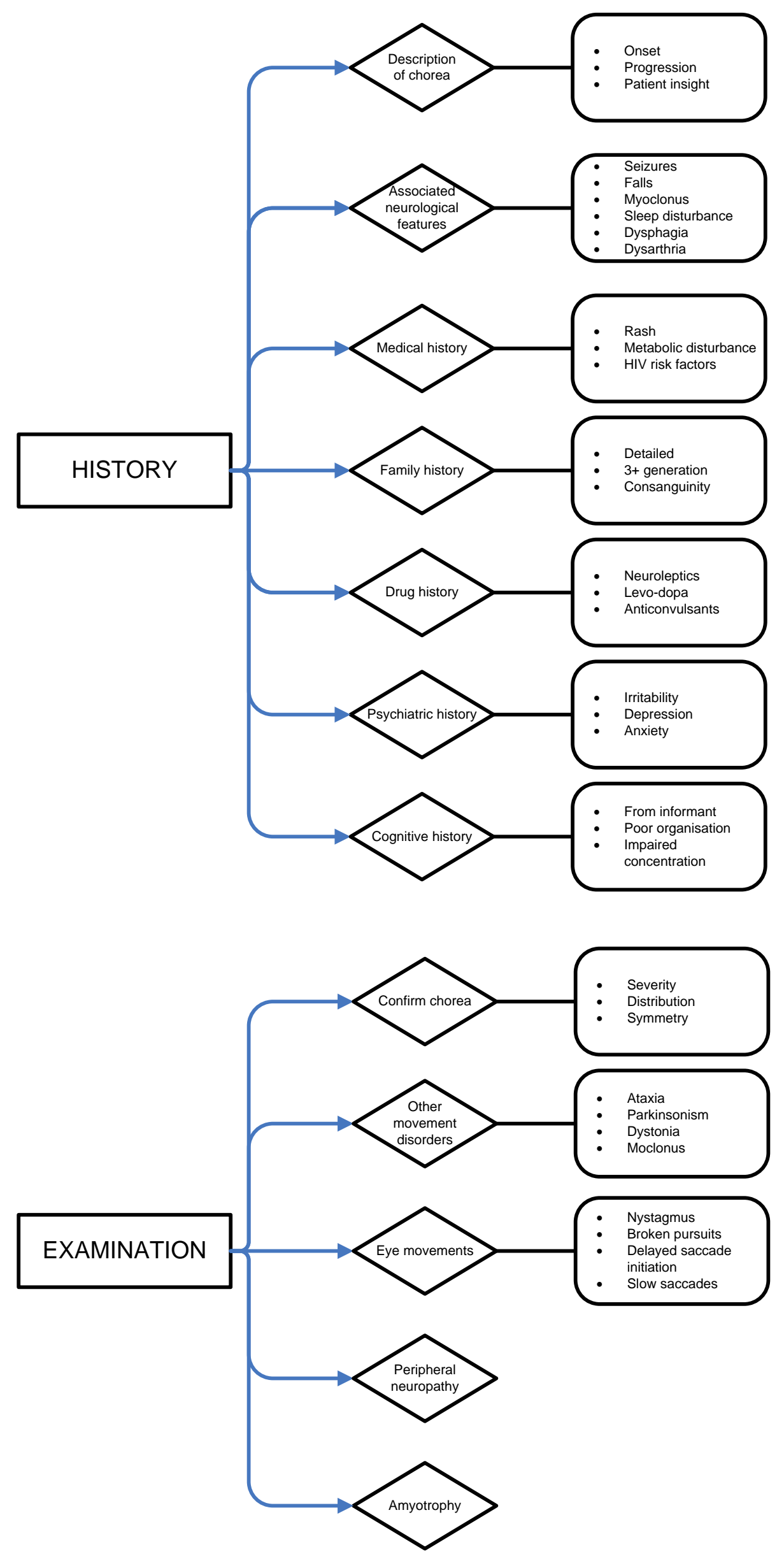




\section{Figure 1. Clinical assessment of the choreic patient}

\section{History}

First, obtain the patient's description of the chorea. A patient with mild chorea may not appear choreic during observation, so it is important to ask about fidgetiness or restlessness. Cognitive impairment may affect insight: patients with HD are notoriously often unaware of their chorea and may deny the existence of obvious movements. ${ }^{8} \mathrm{~A}$ description by a relative or carer is often helpful. Impaired insight makes eliciting the history challenging but can be a useful pointer to the cause of chorea. Most inherited causes of chorea are associated with gradual onset and progression. HD and the other hereditary causes of chorea fall into this category. Rapid onset is more associated with acquired causes, particularly vascular and drug-induced chorea.

Associated neurological features may direct you to the diagnosis. Falls may suggest the presence of another disorder such as pyramidal tract dysfunction, ataxia or peripheral neuropathy. Seizures suggest a diagnosis of DRPLA. Myoclonus is associated with inherited and acquired prion disease. Sleep disturbance, dysarthria and dysphagia are common features of HD.

The past medical history may suggest a cause for the chorea, such as the rash and arthralgia of SLE or symptoms of thyrotoxicosis. It is important to ask about risk factors for HIV transmission. Recent streptococcal infection or history of rheumatic fever or Sydenham's chorea is clearly suggestive.

A detailed family history is essential in the assessment of any choreic patient, but there are several pitfalls. A 3-generation pedigree (i.e. including the subject's parents and grandparents) is the minimum suggested level of enquiry. Autosomal dominant disorders are usually clear from their representation in each generation. Mitochondrial disorders are inherited from the mother and cannot be passed on by males. Autosomal recessive inheritance can be difficult to identify, because there may be no affected relatives within 
several generations. Specific enquiry about consanguineous partnerships, or a distant relative with a similar condition can raise suspicion here.

Genetic aetiology can never be excluded with complete confidence because of the possibilities of non-paternity and new mutations. Conversely, the presence of a positive family history does not exclude an alternative acquired cause. It is therefore important to remain open-minded and, if in doubt, pursue both possibilities. Genetic testing should always be preceded by proper genetic counselling as discussed below.

A detailed drug history may reveal possible causes of chorea (see Table 3) but may also be a confounding factor, for instance in a neuroleptic-treated patient whose psychiatric disturbance is due to HD. Establishing the exact sequence of symptoms and drug treatments is essential.

A cognitive history should be obtained from both the patient and a friend or carer. Particular attention should be paid to the subtle features of frontal-subcortical dementia such as poor organisation and impaired concentration.

A psychiatric history may reveal depression, anxiety or irritability common to HD and its phenocopies.

\section{Examination}

A neurological and general medical examination is essential in the assessment of any patient with new symptoms. Figure 1 highlights key examination findings and Table 5 lists the associations of abnormal findings in the differential diagnosis of chorea.

The first priority is to confirm the presence of chorea. Chorea is usually seen in general observation before formal examination. It is often best noticed out of the corner of the eyefor instance, you may observe a fidgeting foot while the patient is speaking-and may be enhanced by distraction. The first movement abnormality in Huntington's disease is often 
subtle flicking of the fingertips while walking (see online video). Chorea should be distinguished from the list of related disorders in Table 4.

Table 4. Chorea and related hyperkinetic movement disorders

\begin{tabular}{ll}
\hline Disorder & Nature of involuntary movement \\
Chorea & Irregular, seemingly random, semi-directed movements \\
Athetosis & Continuous writhing movements \\
Ballismus & Sudden, vigorous, forceful movements usually involving a whole limb \\
Dystonia & $\begin{array}{l}\text { Sustained contraction of opposing muscle groups causing twisting movements and } \\
\text { abnormal postures }\end{array}$ \\
Myoclonus & Sudden, brief, shock-like movements caused by muscular contraction or inhibition \\
\hline
\end{tabular}

In most cases chorea is bilateral, and symmetrical. Hemichorea is usually due to vascular causes or contralateral basal ganglia lesions (including AIDS-associated chorea).

The presence of other movement disorders is of importance. Cerebellar ataxia is unusual in adult-onset HD and suggests an alternative diagnosis such as spinocerebellar ataxia, Friedreich's ataxia or choreo-acanthocytosis. Dystonia and parkinsonism are present in moderate/advanced $\mathrm{HD}$, juvenile $\mathrm{HD}$ and the Westphal variant. Orolingual dystonia is relatively specific to chorea-acanthocytosis.

Table 5. Abnormal examination findings in the differential diagnosis of chorea

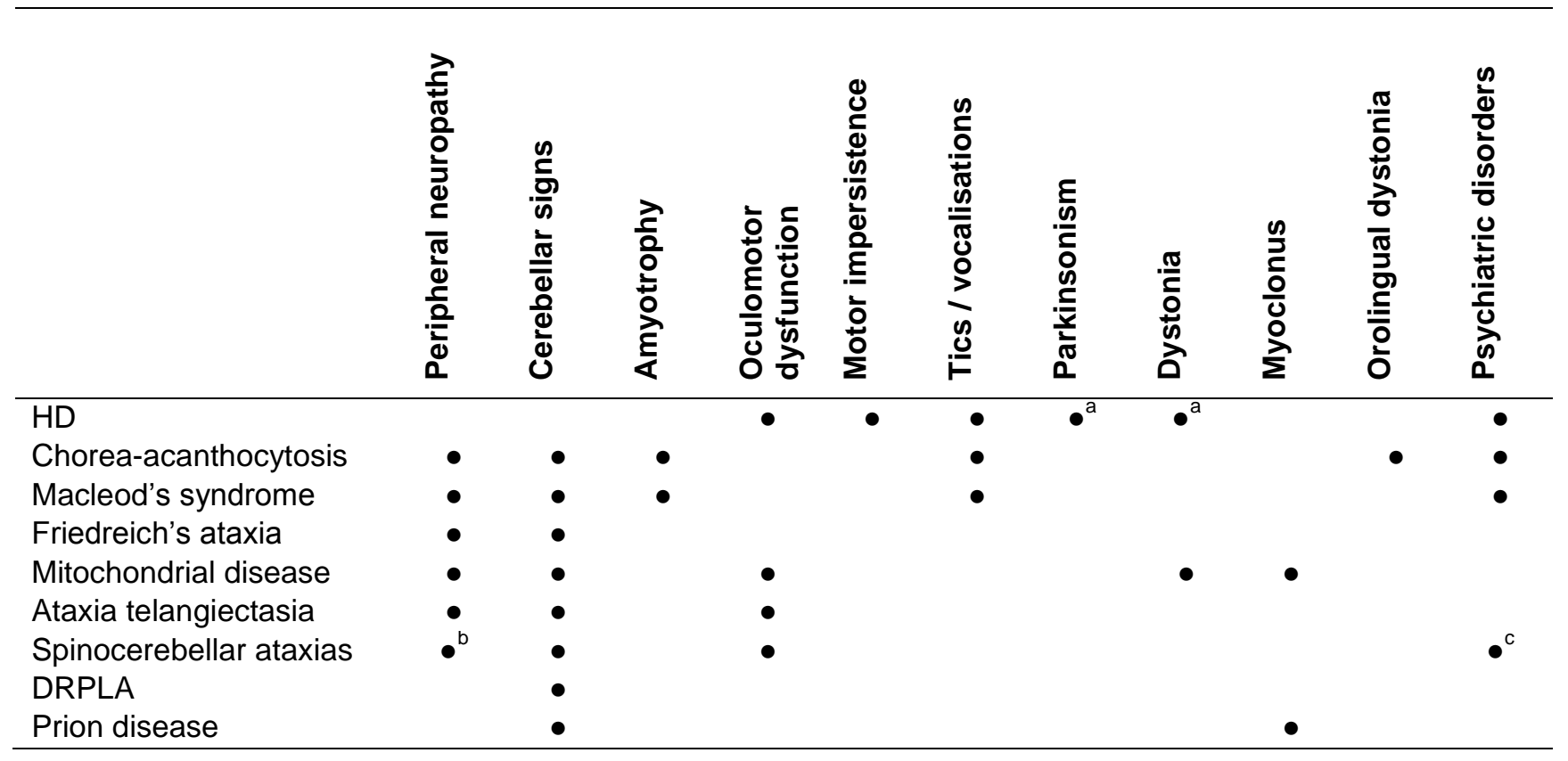




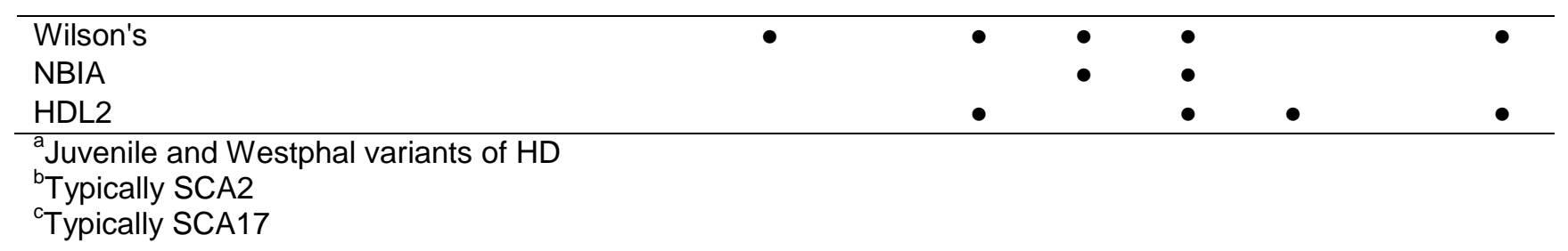

\section{Investigations}

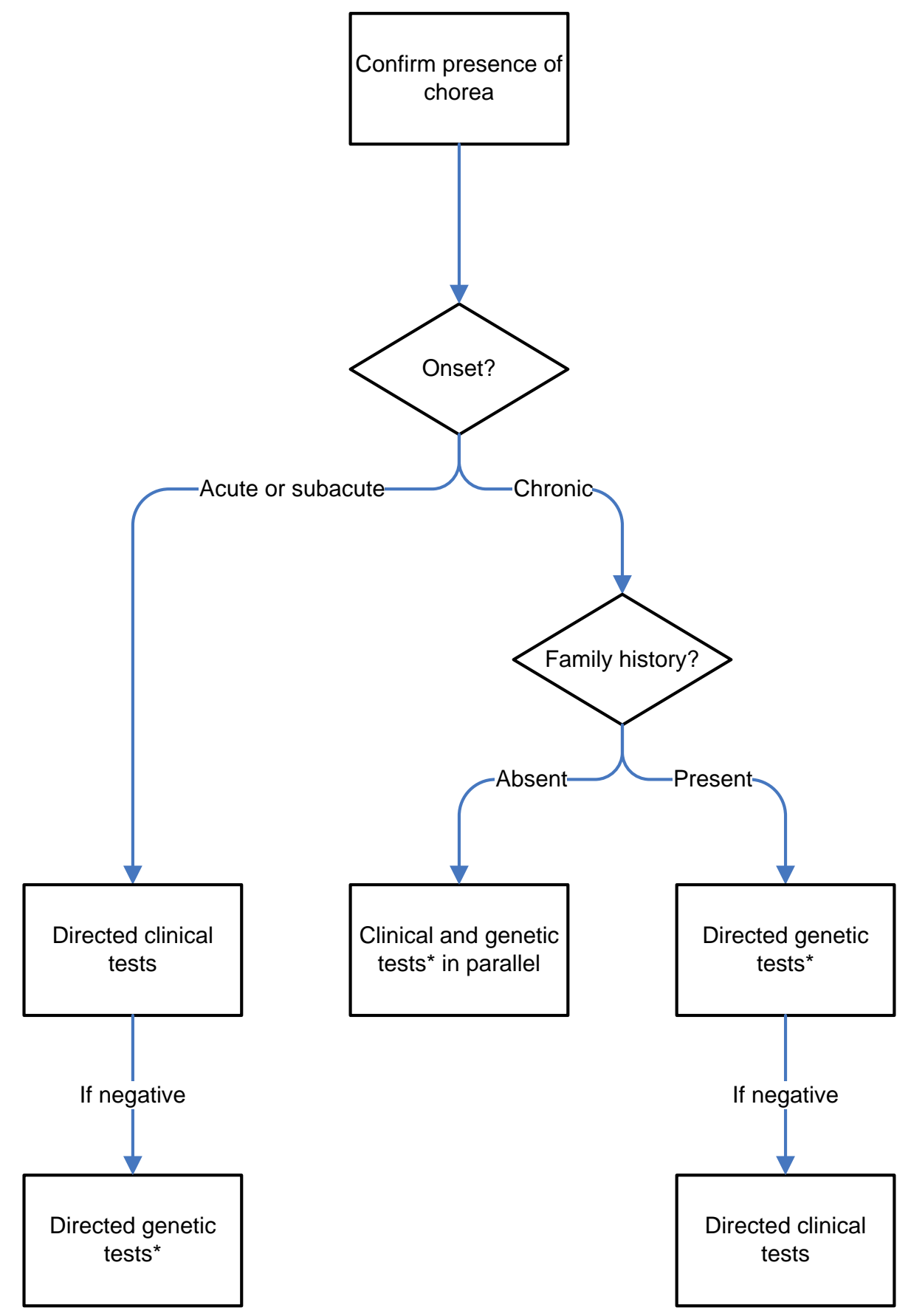

Figure 2. Approach to investigation of the patient with chorea. *Genetic testing should always be accompanied by detailed genetic counselling. 
Investigation of chorea should be directed to the most likely causes (Figure 2). In acute or subacute onset chorea, the diagnosis is most likely to be made on the basis of blood tests and neuroimaging. In chronic or clearly familial cases, genetic testing may render extensive clinical testing unnecessary. In some cases, both approaches may need to be pursued simultaneously. Genetic testing of any patient should always be preceded by genetic counselling.

Table 6. Clinical tests to consider in chorea

\begin{tabular}{ll}
\hline Test & Notes \\
\hline MRI imaging & - Stroke or other focal basal ganglia pathology \\
& - T2* imaging abnormal in iron accumulation \\
& disorders \\
& - FLAIR imaging abnormal in prion disease \\
& - Deep brain T2 hyperintensities in Wilson's disease \\
Full blood count & - Haematocrit may be elevated in PRV \\
Red cell mass & - Sensitive test required for diagnosis of PRV \\
Blood film & - Multiple films necessary to exclude \\
& neuroacanthocytosis \\
Serum caeruloplasmin & - Reduced in Wilson's disease \\
Urinary copper level & - Elevated in Wilson's disease \\
Liver biopsy & - Sometimes required to diagnose Wilson's disease \\
Pregnancy test & - Chorea gravidarum in first trimester \\
HIV test & - Chorea may be presenting feature of AIDS \\
Erythrocyte sedimentation & - Sensitive tests to reveal SLE \\
antinuclear antibody & rate \\
Anti-dsDNA & - Relatively specific to SLE \\
Anticardiolipin and lupus anticoagulant & - Antiphospholipid syndrome is risk factor for chorea \\
Thyroid function tests & - Thyrotoxicosis \\
Anti-streptolysin O titre & - Recent streptococcal infection suggests \\
Antibasal ganglia antibodies & Sydenham's chorea/PANDAS \\
Muscle biopsy & - Associated with post-infective chorea, chorea \\
& gravidarum and OCP-induced chorea \\
Cerebrospinal fluid analysis & - Ragged red fibres or respiratory chain \\
\hline
\end{tabular}

\section{Genetic testing and counselling}

We suggest a structured, sequential approach to genetic testing based on clinical findings (Figure 3). The identification of a new genetic diagnosis has widespread ramifications for the patient and their family members. A positive diagnostic genetic test for HD in a person with no known family history immediately means that all their siblings and children are at $50 \%$ risk of the disease. Genetic testing for any disease has the potential to cause significant distress 
and should not be performed without detailed genetic counselling. Most clinicians are aware of the need for counselling of at-risk individuals for predictive testing, but the fact that a patient is symptomatic does not remove the need to provide detailed counselling, time for reflection and support during and after delivery of the result. There are internationally agreed guidelines for testing of at-risk individuals in HD, which is always performed in a specialist genetics setting. ${ }^{40}$ We summarise guidelines for diagnostic genetic testing in Table 7 , to highlight that, when correctly conducted, the process of genetic counselling requires time, support and expertise that may not be available in the busy general neurology clinic. We therefore recommend if possible that genetic testing be performed through specialist neurogenetics services and supported by lay organisations. ${ }^{40}$

Table 7. Recommended guidelines for diagnostic genetic testing for HD and other autosomal dominant neurodegenerative disorders. Predictive testing in HD is only undertaken in specialist genetic centres.

- Principles of genetic counselling and testing

- Testing for HD should not be part of a routine blood investigation for chorea

- Patient should be encouraged to select a companion to accompany him or her throughout the testing process.

- The patient and their family need to be aware of the implications for them of a positive genetic result

- A follow-up protocol should be agreed

- Strict confidentiality must be observed

- Informed consent signed by neurologist and patient

- Arrangements for counselling and testing

- The patient should be given the result with their chosen companion and not alone

- Result should be delivered as soon as possible after completion of the test, on an agreed date

- The patient has the right to decide not to receive the result or not to be genetically tested.

- A rapid follow-up appointment (within one month) should be arranged after delivery of a positive genetic result

- Information to be given to the patient

- Clinical manifestations of HD, social and psychological implications, genetic aspects, reproductive implications, availability of treatment, and so forth

- Support and information from lay organisations

How test is done

Test cannot predict symptoms, severity or rate of progression

Consequences for the patient, spouse/partner, children and other family members

Socioeconomic consequences (employment, insurance, social security, data security and others) 


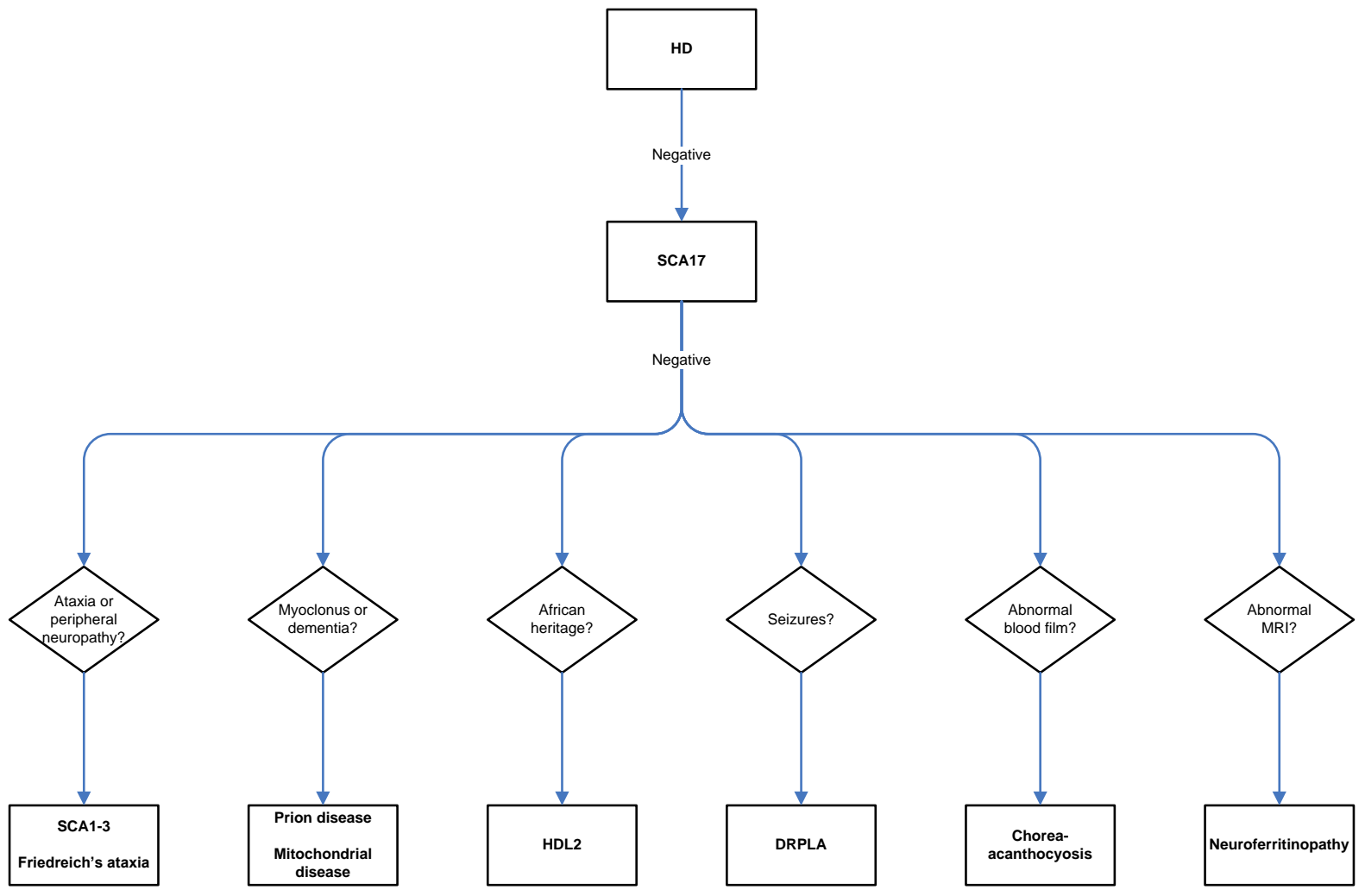

Figure 3. Suggested approach to initial genetic testing in chorea.

\section{Pharmacological management of chorea}

Chorea may cause cosmetic embarrassment or disability, but many patients do not notice the severity of their chorea. Indeed, it is often relatives who request treatment. Anti-choreic medication should be used sparingly as no drug is particularly efficacious, and motor sideeffects may worsen functional disability. Treatment of underlying causes, cognitive and psychiatric features is more important than symptomatic management of chorea. For functionally disabling chorea, sulpiride, olanzapine and risperidone at low doses are useful. Tetrabenazine is a good option, but may cause sedation and depression. ${ }^{12}$

\section{Practice points}

- Chorea may vary in severity from subtle finger-flicking to continuous, violent movements and may overlap with other movement disorders

- A cause is identified in most cases of chorea 
$\circ$ A detailed history and examination will usually reveal associated features that may suggest a diagnosis and direct investigation

- Genetic and acquired causes should be considered and tested for systematically depending on the clinical course and family history

- Huntington's disease is by far the commonest genetic cause of chorea

- Genetic testing for any condition, even in symptomatic patients, should be preceded by detailed, expert counselling that is ideally best provided by a specialist neurogenetics service

\section{Web links}

○ Huntington's Disease Association (www.hda.org.uk)

○K Huntington's Disease Network (www.ukhdn.net)

○ GeneTests medical genetics information (www.geneclinics.org)

\section{References}

1. Krack P. Relicts of dancing mania: The dancing procession of Echternach. Neurology 1999;53(9):2169-72.

2. The Huntington's Disease Collaborative Research Group. A novel gene containing a trinucleotide repeat that is expanded and unstable on Huntington's disease chromosomes. Cell 1993;72(6):971-83.

3. Rubinsztein DC, Leggo J, Coles R, Almqvist E, Biancalana V, Cassiman JJ, et al. Phenotypic characterization of individuals with 30-40 CAG repeats in the Huntington disease (HD) gene reveals HD cases with 36 repeats and apparently normal elderly individuals with 36-39 repeats. Am J Hum Genet 1996;59(1):16-22. 
4. Kremer B, Almqvist E, Theilmann J, Spence N, Telenius H, Goldberg YP, et al. Sexdependent mechanisms for expansions and contractions of the CAG repeat on affected Huntington disease chromosomes. Am J Hum Genet 1995;57(2):343-50.

5. Brandt J, Bylsma FW, Gross R, Stine OC, Ranen N, Ross CA. Trinucleotide repeat length and clinical progression in Huntington's disease. Neurology 1996;46(2):52731.

6. Langbehn DR, Brinkman RR, Falush D, Paulsen JS, Hayden MR. A new model for prediction of the age of onset and penetrance for Huntington's disease based on CAG length. Clinical Genetics 2004;65(4):267-277.

7. Li JL, Hayden MR, Almqvist EW, Brinkman RR, Durr A, Dode C, et al. A genome scan for modifiers of age at onset in Huntington disease: The HD MAPS study. Am J Hum Genet 2003;73(3):682-7.

8. Kremer B. Clinical neurology of Huntington's disease. In: Harper PS, editor. Huntington's disease. Oxford: Oxford Medical Publications, 2002.

9. Lasker AG, Zee DS. Ocular motor abnormalities in Huntington's disease. Vision Res 1997;37(24):3639-45.

10. Craufurd D, Snowden J. Neuropsychological and neuropsychiatric aspects of Huntington's disease. In: Harper PS, editor. Huntington's disease. Oxford: Oxford Medical Publications, 2002.

11. Farrer LA. Suicide and attempted suicide in Huntington disease: implications for preclinical testing of persons at risk. Am J Med Genet 1986;24(2):305-11.

12. Bonelli RM, Hofmann P. A systematic review of the treatment studies in Huntington's disease since 1990. Expert Opin Pharmacother 2007;8(2):141-53.

13. Stevanin G, Fujigasaki H, Lebre A-S, Camuzat A, Jeannequin C, Dode C, et al. Huntington's disease-like phenotype due to trinucleotide repeat expansions in the TBP and JPH3 genes. Brain 2003;126(7):1599-1603. 
14. Margolis RL, Holmes SE, Rosenblatt A, Gourley L, O'Hearn E, Ross CA, et al. Huntington's disease-like 2 (HDL2) in North America and Japan. Annals of Neurology 2004;56(5):670-674.

15. Schols L, Bauer P, Schmidt T, Schulte T, Riess O. Autosomal dominant cerebellar ataxias: clinical features, genetics, and pathogenesis. The Lancet Neurology $2004 ; 3(5): 291-304$.

16. Machado A, Chien HF, Deguti MM, Cancado E, Azevedo RS, Scaff M, et al. Neurological manifestations in Wilson's disease: Report of 119 cases. Mov Disord 2006;21(12):2192-6.

17. Caer M, Viala K, Levy R, Maisonobe T, Chochon F, Lombes A, et al. Adult-onset chorea and mitochondrial cytopathy. Mov Disord 2005;20(4):490-2.

18. Chinnery PF, Crompton DE, Birchall D, Jackson MJ, Coulthard A, Lombes A, et al. Clinical features and natural history of neuroferritinopathy caused by the FTL1 460InsA mutation. Brain 2007;130(Pt 1):110-9.

19. Hartig MB, Hortnagel K, Garavaglia B, Zorzi G, Kmiec T, Klopstock T, et al. Genotypic and phenotypic spectrum of PANK2 mutations in patients with neurodegeneration with brain iron accumulation. Ann Neurol 2006;59(2):248-56.

20. Ueno S, Maruki Y, Nakamura M, Tomemori Y, Kamae K, Tanabe H, et al. The gene encoding a newly discovered protein, chorein, is mutated in chorea-acanthocytosis. Nat Genet 2001;28(2):121-2.

21. Danek A, Walker RH. Neuroacanthocytosis. Current Opinion in Neurology 2005;18(4):386-92.

22. Breedveld GJ, Percy AK, MacDonald ME, de Vries BB, Yapijakis C, Dure LS, et al. Clinical and genetic heterogeneity in benign hereditary chorea. Neurology 2002;59(4):579-84.

23. Schrag A, Quinn NP, Bhatia KP, Marsden CD. Benign hereditary chorea-entity or syndrome? Mov Disord 2000;15(2):280-8. 
24. Hanna MG, Davis MB, Sweeney MG, Noursadeghi M, Ellis CJ, Elliot P, et al. Generalized chorea in two patients harboring the Friedreich's ataxia gene trinucleotide repeat expansion. Mov Disord 1998;13(2):339-40.

25. Piccolo I, Defanti CA, Soliveri P, Volontè MA, Cislaghi G, Girotti F. Cause and course in a series of patients with sporadic chorea. Journal of Neurology 2003;250(4):429435.

26. Cardoso F, Silva CEAP, Mota CCC. Chorea in fifty consecutive patients with rheumatic fever. Movement Disorders 1997;12(5):701-703.

27. Ghika-Schmid F, Ghika J, Regli F, Bogousslavsky J. Hyperkinetic movement disorders during and after acute stroke: The Lausanne Stroke Registry. Journal of the Neurological Sciences 1997;146(2):109-116.

28. Church AJ, Cardoso F, Dale RC, Lees AJ, Thompson EJ, Giovannoni G. Anti-basal ganglia antibodies in acute and persistent Sydenham's chorea. Neurology 2002;59(2):227-31.

29. Cardoso F, Maia D, César M, Cunningham QS, Valença G. Treatment of Sydenham chorea with corticosteroids. Movement Disorders 2003;18(11):1374-1377.

30. Perlmutter SJ, Leitman SF, Garvey MA, Hamburger S, Feldman E, Leonard HL, et al. Therapeutic plasma exchange and intravenous immunoglobulin for obsessivecompulsive disorder and tic disorders in childhood. The Lancet 1999;354(9185):1153-1158.

31. Marschitz I, Rodl S, Gruber-Sedlmayr U, Church A, Giovannoni G, Zobel G, et al. Severe chorea with positive anti-basal ganglia antibodies after herpes encephalitis. $J$ Neurol Neurosurg Psychiatry 2007;78(1):105-107.

32. Jain KK. Drug-induced movement disorders. Drug-induced neurological disorders. Seattle: Hogrefe \& Huber, 1996.

33. Miranda M, Cardoso F, Giovannoni G, Church A. Oral contraceptive induced chorea: another condition associated with anti-basal ganglia antibodies. J Neurol Neurosurg Psychiatry 2004;75(2):327-328. 
34. Cervera R, Asherson RA, Font J, Tikly M, Pallares L, Chamorro A, et al. Chorea in the antiphospholipid syndrome. Clinical, radiologic, and immunologic characteristics of 50 patients from our clinics and the recent literature. Medicine (Baltimore) 1997;76(3):203-12.

35. Isaacs JD, Rakshi J, Baker R, Brooks DJ, Warrens AN. Chorea associated with thyroxine replacement therapy. Mov Disord 2005;20(12):1656-7.

36. Tse W, Cersosimo MG, Gracies JM, Morgello S, Olanow CW, Koller W. Movement disorders and AIDS: a review. Parkinsonism Relat Disord 2004;10(6):323-34.

37. Dike GL. Chorea gravidarum: a case report and review. Md Med J 1997;46(8):436-9.

38. Nazabal ER, Lopez JM, Perez PA, Del Corral PR. Chorea disclosing deterioration of polycythaemia vera. Postgrad Med J 2000;76(900):658-659.

39. Cao M, Olsen RJ, Zu Y. Polycythemia vera: new clinicopathologic perspectives. Arch Pathol Lab Med 2006;130(8):1126-32.

40. International Huntington Association and World Federation of Neurology Research Group on Huntington's Chorea. Guidelines for the molecular genetics predictive test in Huntington's disease. Neurology 1994;44(8):1533-6. 